\title{
A importância do exame radiográfico torácico na abordagem de animais portadores de neoplasias
}

\section{Importance of thoracic radiography in the approach of animals with neoplasia}

\author{
Tatiana Soave ${ }^{1 *}$; Daniela Paukowiski de Sousa ${ }^{1}$; Kleber Moreno ${ }^{2}$; Suely N. E. Beloni ${ }^{3}$; \\ Janis R. M. Gonzáles ${ }^{3}$; Claudia C. Boselli Grotti ${ }^{4}$; Antonio Carlos Faria dos Reis ${ }^{5}$
}

\section{Resumo}

O objetivo deste trabalho foi avaliar a importância do exame radiográfico torácico em cães e gatos com neoplasias de diversas origens e localizações, excetuando neoplasias mamárias. Foram estudados 54 animais com suspeita de metástase pulmonar e/ou neoplasia pulmonar primária - 49 cães (91\%) e cinco felinos ( $9 \%$ ); sendo 28 (52\%) fêmeas ( 3 felinas) e 26 (48\%) machos (2 felinos) - atendidos nos Projetos de Extensão em Medicina Torácica e Oncologia do Hospital Veterinário/UEL, durante o ano de 2005. As neoplasias mamárias, não foram inclusas neste trabalho. Dos 54 animais, seis (11\%) apresentaram exames radiográficos com evidência de metástase pulmonar, sendo um felino. Quatro animais $(8 \%)$ tiveram exame radiográfico compatível com neoplasia pulmonar primária. Esses resultados indicam a importância do exame radiográfico torácico na abordagem de animais com neoplasias, para descartar a possibilidade de metástase pulmonar independente da origem neoplásica e, a necessidade de monitoramento radiográfico nos animais sem sinais radiográficos de metástase pulmonar.

Palavras-chave: Metástase, neoplasia, pulmão, cães, gato

\begin{abstract}
The aim of the present study was to evaluate the importance of the thoracic radiography in dogs and cats with neoplasias of diverse origins and localizations, excepting mammary neoplasm. It was studied 54 animals on suspicion of pulmonary metastase and/or primary lung tumors- 49 dogs $(91 \%)$ and five cats ( $9 \%)$; being $28(52 \%)$ female ( 3 cats) and $26(48 \%)$ male ( 2 cats) - attended by Thoracic and Oncology Medicine Services in the Veterinary Hospital/UEL, in 2005. The mammary neoplasias were not included in this work. From the 54 animals, six $(11 \%)$ presented radiography examinations with evidence of pulmonary metastase, being one cat. Four animals $(8 \%)$ had compatible radiography examination with primary pulmonary neoplasia. These results indicate the importance of the thoracic radiography in the approach of animals with neoplasia, to exclude the possibility of pulmonary metastase independent from the neoplasm origin and the necessity of radiographic attendance to the animals without radiographic signals of pulmonary metastase.
\end{abstract}

Key words: Metastase, neoplasia, pulmonary, dogs, cats

\footnotetext{
1 Médica Veterinária. End.: Rua Ibiporã, 686-A, Jardim Santo Antônio, Londrina-PR, CEP 86060-510. E-mail: datsoave@yahoo.com.br

2 Médico Veterinário, Doutorando do Programa de Pós-graduação em Ciência Animal, UEL.

3 Professor do Departamento de Clínicas Veterinárias, CCA, UEL.

4 Médica Veterinária, Técnica de nível Superior do Laboratório de Patologia Animal, Departamento de Medicina Veterinária Preventiva, CCA, UEL.

5 Professor do Departamento de Medicina Veterinária Preventiva, CCA, UEL.

* Autor para correspondência
} 
O exame radiográfico torácico é importante na abordagem de animais com neoplasias, pois possibilita o diagnóstico de metástase pulmonar, além de auxiliar na coleta de amostras de tecido pulmonar ou de massas intratorácicas, para exames histopatológicos e citológicos.

As neoplasias primárias de pulmão em cães são raras, comparadas com humanos, correspondendo a apenas $1 \%$ dos cânceres diagnosticados. Em gatos, a incidência é ainda menor (WITHROW, 2001). A maioria dos tumores pulmonares primários são carcinomas, sendo os adenocarcinomas de origem bronquial ou epitelial alveolar os mais comuns (70 a $80 \%)$ e os de células escamosas os menos freqüentes. Outras neoplasias pulmonares primárias menos freqüentes são os tumores mesenquimais, como os osteossarcomas. As neoplasias pulmonares benignas são raras (HAWKINS, 2004).

Metástases de tumores primários pulmonares em animais são comuns, devido à alta malignidade destes tumores e ocorrem em 100\% dos carcinomas epidermóides, 90\% dos carcinomas anaplásicos e em $50 \%$ dos adenocarcinomas (FOSSUM; ROGERS, 1998). Em felinos a incidência de metástase é de 75\% (WITHROW, 2001). As células neoplásicas podem ser disseminadas para outras áreas pulmonares via corrente sangüínea, linfática, aérea e transpleural. As áreas mais comuns de metástases de tumores pulmonares primários são os próprios pulmões, linfonodos brônquicos e mediastinais (FOSSUM; ROGERS, 1998; COUTO, 1998). As metástases podem ainda envolver órgãos extratorácicos como o sistema nervoso central (SNC), ossos longos e órgãos intra-abdominais (HAWKINS, 2004). Tanto o câncer pulmonar humano como o de animais, parece ter propensão a invadir o SNC (WITHROW, 2001).

Animais com neoplasia pulmonar apresentam sinais clínicos variados, que podem ser confundidos com sinais de doenças cardíacas ou intratorácicas, como: tosse crônica, intolerância ao exercício, emagrecimento progressivo, taquipnéia e/ou dispnéia.
A angústia respiratória pode estar associada a pneumotórax, efusão pleural, obstrução/compressão de vias aéreas (HAWKINS, 2004; COUTO, 1998). Os felinos apresentam sinais pouco específicos e que dificilmente estão correlacionados com o tumor pulmonar, como: letargia, anorexia, perda de peso, dispnéia e/ou taquipnéia e tosse não produtiva (COUTO; HAMMER, 1994). Animais com neoplasia pulmonar primária podem também apresentar hemoptise, embora este seja um sinal raro (NELSON; SELLON, 2005).

Os animais acometidos por neoplasia pulmonar podem apresentar síndromes paraneoplásicas, com as seguintes alterações clínicas ou laboratorias: hipercalcemia, anorexia, febre, emagrecimento, paraplegia, neuromiopatia, caquexia e secreção de hormônios adrenocorticotróficos (FOSSUM; ROGERS, 1998). Dentre as síndromes paraneoplásicas, a osteopatia hipertrófica, cursando com claudicação é descrita com maior freqüência em cães, sendo rara em gatos (HAWKINS, 2004; FILGUEIRAS et al., 2002). No entanto, tais síndromes são bem mais freqüentes em humanos (MACEWEN; KHANNA; RADINSKY, 2001). Alterações clínicas secundárias às síndromes paraneoplásicas como: claudicação, efusão abdominal, alterações neurológicas, entre outras, podem estar presentes dependendo da localização do tumor (NELSON; SELLON, 2005).

As neoplasias pulmonares metastáticas são mais comuns do que as primárias, pois é no pulmão que se localiza o principal sistema capilar carreador da maioria das células neoplásicas circulantes (HAWKINS, 2004). Os osteossarcomas, carcinomas de células escamosas, melanomas, hemangiossarcomas e, os carcinomas mamários em cães e gatos são os principais causadores de metástase pulmonar, porém qualquer tumor maligno pode produzir metástases (CAROTHERS, 1998).

Os sinais clínicos apresentados por animais portadores de doença pulmonar metastática são semelhantes àqueles dos tumores pulmonares 
primários, e podem também ser confundido com outras doenças torácicas (NELSON; SELLON, 2005).

As neoplasias pulmonares primárias, ao exame radiográfico, apresentam padrões muito variáveis, incluindo lesões expansivas, circunscritas isoladas, consolidação lobar, massas circunscritas múltiplas e envolvimento difuso. Cavitações de lesões expansivas, linfonodomegalia hilar e calcificação de lesões, também podem ser observadas; porém observa-se com maior freqüência uma densidade nodular solitária em lobos caudais (LAMB, 2002).

As neoplasias pulmonares metastáticas normalmente apresentam padrão intersticial reticular com múltiplos nódulos em toda a extensão dos campos pulmonares; podendo também variar desde o padrão intersticial nodular bem definido ao mal definido, associado ou não ao quadro alveolar. Estes padrões radiográficos podem ser confundidos com infecções (especialmente fúngicas), reações a corpo estranho, hipersensibilidade, doenças imunomediadas e torção de lobo pulmonar (BURK; ACKERMAN, 1996). Também pode ser detectado envolvimento de linfonodo regional ou ossos (NYKAMP; SCRIVANI; DYKES, 2002).

Nos exames radiográficos somente são detectados nódulos pulmonares maiores que 4-5 mm; sendo que a sensibilidade das radiografias torácicas em detectar metástase pulmonar está estimada em 65 a 97\%, desde que sejam realizadas no mínimo duas projeções (lateral direita e ventrodorsal/ dorsoventral) (LAMB, 2002). Porém, o exame tem maior fidedignidade, quando se faz três projeções (laterais direito e esquerdo e ventrodorsal/ dorsoventral) (BAEZ; SORENMO, 2004).

Líquidos pleurais, lavados traqueais e broncoalveolares podem ser avaliados citologicamente; porém deve-se fazer a diferenciação entre as células neoplásicas e células epiteliais hiperplásicas presentes na resposta inflamatória grave (MELLO; FERREIRA, 2003). Biópsia transtorácicas ou transbronquiais pode ser efetuada, respectivamente, em lesões próximas à parede torácica ou envolvendo vias aéreas principais. A toracotomia, também pode ser usada como método diagnóstico, a qual muitas vezes é terapêutica, porém consiste em um procedimento bastante invasivo. Outros exames são a tomografia computadorizada e a toracoscopia (NELSON; SELLON, 2005).

Na necropsia, os tumores pulmonares primários aparecem normalmente como massas únicas, invasivas ou expansivas, com coloração e textura variáveis. As neoplasias pulmonares secundárias são usualmente múltiplas, de tamanhos variáveis e, de acordo com a distribuição podem ser nodulares, difusas ou radiadas (LOPEZ, 1998).

Como os sinais clínicos de neoplasias e metástases pulmonares podem ser confundidos com os de doenças cardiorespiratórias, e frente à dificuldade e inespecificidade de outros exames complementares, o presente levantamento tem por objetivo, analisar a importância do exame radiográfico torácico na abordagem de animais com neoplasia.

Foram analisadas radiografias de animais da espécie canina e felina com suspeita de metástase pulmonar e/ou neoplasia pulmonar primária, atendidos nos Projetos de Extensão em Medicina Torácica e Oncologia do Hospital Veterinário/UEL, durante o ano de 2005.

O levantamento compreendeu as fichas de encaminhamento à radiologia de animais com suspeita clínica de metástase pulmonar e/ou neoplasia pulmonar primária. Ainda com base na ficha de encaminhamento à radiologia foram coletados dados como: sexo, idade, raça, localização da neoplasia primária, sinais clínicos e tempo de evolução. Após este levantamento inicial foram avaliados os laudos radiográficos destes animais.

Os exames radiográficos foram realizados conforme a possibilidade de imobilização: decúbito lateral direito ou esquerdo nas projeções laterolaterais e decúbito dorsal na projeção ventrodorsal. As técnicas radiográficas basearam-se em método que relaciona a quilovoltagem e miliamperagem, segundo 
a espessura da região a ser radiografada. As radiografias foram escolhidas conforme a suspeita clínica de neoplasia pulmonar primária ou metastática, tendo como base os laudos radiográficos, que evidenciaram tal suspeita ou não.

Após o levantamento radiográfico, foram analisados os laudos dos exames anatomopatológicos referentes aos animais selecionados via exame radiográfico torácico.

Neste estudo não foram incluídas as neoplasias mamárias, pois o objetivo foi avaliar a ocorrência de metástases pulmonares em animais portadores de outros tipos de neoplasias, excetuando-se as mamárias.

Foram estudados 54 animais com suspeita de metástase pulmonar e/ou neoplasia pulmonar primária - 49 caninos (91\%) e cinco felinos (9\%); sendo 28
(52\%) fêmeas (três felinas) e 26 (48\%) machos (dois felinos). A idade variou entre 3 e 15 anos, com maior ocorrência na faixa de 6 a 10 anos, representada por 24 animais (44\%).

Entre os caninos, 18 eram sem raça definida (SRD). E, as raças encontradas foram: rottweiler $(n=6)$, boxer $(n=5)$ e poodle $(n=4)$, representando o maior número de animais em cada raça. Outras raças encontradas foram: cocker, pinsher, teckel, collie, dog alemão, fila, husky, akita, pastor alemão. Dentre os felinos, quatro eram SRD e 1 persa.

As alterações clínicas referentes ao sistema respiratório encontradas estão na Tabela 1. Outros sinais clínicos encontrados foram: perda de peso, intolerância a exercícios, anorexia, letargia, dor abdominal e aumento de linfonodos, que tanto no presente estudo como na literatura podem ser os únicos sinais em uma doença pulmonar neoplásica.

Tabela 1. Alterações clínicas encontradas nos animais com suspeita de metástase pulmonar ou neoplasia pulmonar primária

\begin{tabular}{lcc}
\hline Alterações clínicas & Número de animais & \% de animais \\
\hline Áreas de silêncio pulmonar & 3 & 5 \\
Dispnéia mista e/ou expiratória & 3 & 5 \\
Efusão pleural & 1 & 2 \\
Hemoptise & 1 & 2 \\
Hipofonese & 1 & 2 \\
Secreção nasal serosa & 1 & 2 \\
Sons cardíacos abafados & 1 & 2 \\
Tosse & 5 & 9 \\
\hline
\end{tabular}

O tempo de evolução das alterações clínicas, segundo os proprietários, foi bastante variável, de uma semana a dois anos, incluindo aqueles que não souberam informar. Dos 54 animais, seis (11\%) apresentaram exames radiográficos com evidência de metástase pulmonar, sendo um felino e, quatro
(8\%), apresentaram exame radiográfico compatível com neoplasia pulmonar primária. Os $43(80 \%)$ restantes não apresentaram evidências radiográficas de nódulos pulmonares. Apenas uma das radiografias encontrava-se com técnica insatisfatória (mesmo assim, este animal foi mantido no estudo, pois foi submetido à necropsia). 
As neoplasias primárias estavam localizadas em diferentes regiões anatômicas, sendo 13 (24\%) em cabeça ou região cervical, 13 (24\%) em tronco, seis (11\%) em membros, 12 (22\%) em tórax, seis (11\%) em locais diversos e quatro (8\%) animais apresentavam linfonodomegalia generalizada.

Dentre o número total de animais, 24 (44\%) foram submetidos ao exame citológico, 14 (26\%) ao exame histopatológico e 16 (30\%) animais não realizaram nenhum tipo de exame para determinar o tipo de neoplasia, devido a não autorização dos proprietários. Em 13 (34\%) dos animais submetidos ao exame cito ou histopatológico o exame foi inconclusivo, ou devido à escassez de material ou devido a processo inflamatório local no momento da coleta, não sendo possível determinar a origem neoplásica. Estes animais foram mantidos, pois os exames radiográficos destes foram selecionados inicialmente através das suspeitas clínicas e dos exames radiográficos.

As neoplasias encontradas, segundo a sua origem celular, foram: doze (48\%) em células mesenquimais, seis $(24 \%)$ em células epiteliais, seis (24\%) em células redondas e uma (4\%) neoplasia maligna de origem indeterminada (segundo o laudo citológico, sendo que o material não enviado para o exame histopatológico devido a não autorização pelo proprietário do animal). As neoplasias encontradas estão descritas na Tabela 2.

Tabela 2. Classificação da neoplasia segundo a origem celular

\begin{tabular}{lcc}
\hline Tipo celular da neoplasia & Número de animais & Metástase pulmonar \\
\hline Carcinoma bronquioalveolar & 1 & não \\
Carcinoma de células estratificadas & 1 & não \\
Carcinoma de epitélio respiratório & 1 & sim \\
Carcinossarcoma & 1 & não \\
Emondroma pulmonar & 1 & não \\
Fibrohistocitoma maligno & 1 & sim \\
Fibrossarcoma pulmonar & 1 & não \\
Hemangiossarcoma & 1 & não \\
Histiocitose maligna & 1 & não \\
Linfo ma & 4 & não \\
Mastocitoma & 1 & não \\
Melanoma & 1 & não \\
Osteocondroma & 1 & não \\
Sarcoma histiocítico & 1 & não \\
Sarcoma ocular pós-traumático & 1 & não \\
Sarcoma pouco diferenciado & 1 & sim \\
\hline
\end{tabular}

Dentre os quatro animais com linfoma, três eram cutâneos e um multicêntrico. Os outros três animais com imagem radiográfica compatível com metástase pulmonar e um com imagem de neoplasia pulmonar primária, não foram submetidos a exame citológico ou histopatológico, devido à não autorização dos proprietários.
Este estudo mostrou que $11 \%$ dos animais com neoplasias localizadas em diferentes regiões apresentaram metástases pulmonares; valores semelhantes encontrados na década de 80 para os tumores mamários (WITHROW; SUSANECK, 1986), e em estudo mais recentes tal ocorrência de 
metástase pulmonar secundária a neoplasia mamária aumentou para 25-50\% (HEDLUND, 2002). Estes dados mostram a importância de uma investigação torácica rotineira em animais com neoplasia independente da localização anatômica.

O exame radiográfico por ser um procedimento diagnóstico rápido, simples, não invasivo, de baixo custo e com resultado imediato, deve ser realizado em todo animal portador de neoplasia, antes de qualquer outro exame para determinar a origem tumoral, pois uma vez não evidenciada metástase pulmonar, o clínico pode dispor de métodos diagnósticos para o estadiamento da neoplasia e assim estabelecer o tratamento mais indicado àquele paciente. E quando houver evidência de doença metastática pulmonar auxilia no estabelecimento prognóstico e no esclarecimento ao proprietário. Sendo que muitas vezes a doença metastática é um fator limitante no tratamento da neoplasia primária (HAWKINS, 2004).

Este levantamento retrospectivo mostrou que, como descrito na literatura (WITHROW, 2001), animais de meia idade a idosos (6 a 10 anos) são mais comumente acometidos por neoplasias e, que não há predisposição sexual.

Apesar do maior número de animais ser sem raça definida, dentre os com raça definida, as raças boxer e rottweiler foram mais acometidas, como também já descrito por outros autores (FOSSUM; ROGERS, 1998). O maior número de animais neste estudo foi de SRD ( $\mathrm{n}=22$ ), provavelmente pelo tipo de população canina e felina atendidas no HV/UEL.

Segundo estudos, aproximadamente $25 \%$ dos cães com tumor pulmonar não apresentam sinais clínicos ligados ao trato respiratório (FOSSUM; ROGERS, 1998), assim como foram encontrados muitos animais no presente estudo.

Nos tumores primários de pulmão, o exame radiográfico também é importante, pois facilita a exclusão de outras doenças torácicas, delimita a área comprometida, direcionando o clínico na realização de procedimento que permitam estabelecer o diagnóstico definitivo através do exame citológico e/ ou histopatológico (HAWKINS, 2004).

Esses resultados indicam a importância do exame radiográfico torácico na abordagem de animais com neoplasias, para descartar a possibilidade de metástase pulmonar independente da origem neoplásica.

O exame radiográfico torácico é também importante no monitoramento de animais sem sinais de metástase pulmonar, após tratamento quimioterápico e / ou cirúrgico da neoplasia, devendo ser realizado em média a cada 1 a 3 meses, dependendo do tipo de neoplasia e do estadiamento clínico (CAROTHERS, 1998).

\section{Agradecimentos}

Agradeço aos professores e pós-graduandos que me auxiliaram na realização deste trabalho.

\section{Referências}

BAEZ, J. L.; SORENMO, K. U. Pulmonary and bronchial neoplasia. In: KING L. G. Respiratory disease in dogs and cats. Saint Louis: Saunders, 2004. p. 508-515.

BURK, R. L.; ACKERMAN, N. The thorax. In: Small animal radiology and ultrasonography. 2.ed. Philadelphia: Saunders, 1996. p. 158-188.

CAROTHERS, M. Neoplasia respiratória. In: BICHARD S. J.; SHERDING R. G. Clínica de pequenos animais. São Paulo: Roca, 1998. p. 663-665.

COUTO, C. G. Distúrbios do parênquima pulmonar. In: NELSON, R. W.; COUTO C. G. Medicina interna de pequenos animais. 2.ed. Rio de Janeiro: Guanabara Koogan, 1998. p. 241-243.

COUTO, C. G.; HAMMER, A. S. Oncology. In: SHERDING, R. G. The cat diseases and clinical management. 2.ed. Philadelphia: Saunders, 1994. p. 755-762, 807-808.

FILGUEIRAS, R. R.; SILVA, J. C. P.; VILÓRIA, M. I. V.; ODENTHAL, M. E.; DUARTE, T. S.; LAVOR, M. S. L. Osteopatia hipertrófica em cão - relato de caso. Revista Clínica Veterinária, São Paulo, v. 7, n. 36, p. 28-31, 2002. 
FOSSUM, T. W.; ROGERS, K. S. Sistema respiratório: oncologia. In: SLATTER, D. Manual de cirurgia de pequenos animais. 2.ed. São Paulo: Manole, 1998. v. 2, p. 2635-2641.

HAWKINS, E. C. Doenças do parênquima pulmonar. In: ETTINGER, S. J. Tratado de medicina interna veterinária. 5.ed. Rio de Janeiro: Guanabara Koogan, 2004. v. 2, p. 1131-1138.

HEDLUND, C. S. Cirurgia do sistema reprodutor e genital. In: FOSSUM, T. W. Cirurgia de pequenos animais. São Paulo: Roca, 2002. p. 596-601.

LAMB, C. R. The canine and feline lung. In: THRALL, D. E. Text book of veterinary diagnostic radiology. 4.ed. Philadelphia: Saunders, 2002. p. 431-447.

LOPEZ, A. Sistema respiratório. In: CARLTON, W. W.; MCGAVIN, M. D. Patologia veterinária especial. 2.ed. Porto Alegre: Artes Médicas Sul, 1998. p. 188-190.

MACEWEN, E. G.; KHANNA, C.; RADINSKY, R. Tumor biology. In: WITHROW, S. J.; MACEWEN, E. G. Small animal clinical oncology. 3.ed. Philadelphia: Saunders, 2001.p. 18-34.
MELLO, M. F. V.; FERREIRA, A. M. R. Análise citológica do líquido de lavagem broncoalveolar para diagnóstico das doenças pulmonares: revisão. Revista Clínica Veterinária, São Paulo, v. 8, n. 42, p. 52-58, 2003.

NELSON, O. L.; SELLON R. K. Pulmonary parenchymal disease. In: ETTINGER, S. J. TexBook of veterinary internal medicine. 6.ed. St Louis: Saunders, 2005. p. 12411247.

NYKAMP, S. G.; SCRIVANI, P. V.; DYKES, N. L. Radiographic signs of pulmonary disease: an alternative approach. Compendium on Continuing Education for the Practicing Veterinarian, Princeton, v. 24, n. 1, p. 25-35, 2002.

WITHROW, S. J. Tumor of the respiratory system. In: WITHROW S. J.; MACEWEN E. G. Small animal clinical oncology. 3.ed. Philadelphia: Saunders, 2001. p. 361-369.

WITHROW, S. J.; SUSANECK, S. J. Tumors of the canine female reproductive tract. In: MORROW, D. A. Current therapy in theriogenology. 2.ed. Philadelphia: Saunders, 1986. p. 521-523. 
